\title{
Dos registros de Agaricales (Basidiomycota) exóticos en bosques nativos de la Patagonia argentina.
}

\author{
(Two records of exotic Agaricales (Basidiomycota) in native forests \\ of the Argentinean Patagonia)
}

\begin{abstract}
Romano Gonzalo ${ }^{\text {ab*}}$, Francisco Kuhar ${ }^{\text {abc }}$
aUniversidad Nacional de la Patagonia San Juan Bosco. Esquel, Chubut, Argentina. ${ }^{b}$ Consejo Nacional de Investigaciones Científicas y Técnicas ${ }^{\mathrm{c} C e n t r o ~ d e ~ I n v e s t i g a c i o ́ n ~ y ~ E x t e n s i o ́ n ~ F o r e s t a l ~ A n d i n o ~ P a t a g o ́ n i c o ~}$

*Autor para correspondencia: gonza.romano@gmail.com
\end{abstract}

RECIBIDO:27 de Mayo de 2017

APROBADO:21 de Junio de 2017

DOI: 10.22370/bolmicol.2017.32.1.660

LOS AUTORES DECLARAN NO TENER CONFLICTO DE INTERESES

Palabras claves: Agaricales; bosque; invasión; micorriza; plantación.

Key words: Agaricales; forest; invasion; mycorrhiza; plantation.

\section{RESUMEN}

Inocybe sindonia (Fr.) P. Karst. y Amanita rubescens Pers. son especies típicamente asociadas a bosques de Pseudotsuga menziesii (Mirb.) Franco y Pinus radiata D. Don respectivamente. Se reporta por primera vez su presencia en Argentina en bosques de Nothofagus de la Patagonia andina como resultado de la invasión de especies forestales introducidas como recurso maderero. $I$. sindonia fue colectado en bosques de $N$. dombeyi (Mirb.) Oerst. y A. rubescens en bosques de $N$. pumilio (Poepp. \& Endl.) Krasser en áreas protegidas con invasión evidente de coníferas exóticas.

\section{ABSTRACT}

Inocybe sindonia (Fr.) P. Karst. and Amanita rubescens Pers. are species typically associated to Pseudotsuga menziesii (Mirb.) Franco and
Pinus radiata D. Don respectively. Both species have been introduced in South America together with timber species plantations. Basidiomes of $I$. sindonia were found occurring under Nothofagus dombeyi (Mirb.) Oerst., while basidiomes of $A$. rubescens were found under N. pumilio (Poepp. $\&$ Endl.) Krasser in protected areas that are under invasion of exotic conifers.

\section{INTRODUCCIÓN}

Los bosques de Nothofagus de la Patagonia andina han sufrido el impacto del uso forestal, ganadero y la introducción de especies madereras exóticas invasivas tales como Pseudotsuga menziesii y Pinus radiata. Dado que tanto las especies del género Nothofagus como las coníferas mencionadas son ectomicorrícicas, las especies de hongos involucradas en la interacción forman parte del fenómeno de la invasión. De tal mane- 
ra, las plantaciones son fuente de inóculo esporal que facilitan la subsiguiente invasión de especies exóticas en la patagonia ${ }^{1}$.

El estrato superior de los bosques andino patagónicos está formado por especies del género Nothofagus, con N. pumilio como su mayor representante. Se trata de la especie nativa de mayor importancia para la región, no sólo por su valor ecológico sino también por su valor histórico para la industria forestal. Después de Tierra del Fuego, la provincia de Chubut es la que tiene mayor superficie de bosques de lenga en la Argentina ${ }^{2}$. Con el transcurso de los años, la industria maderera ha centrado la explotación de coníferas, lo que ha llevado a un proceso de aumento del número $\mathrm{y}$ tamaño de las plantaciones, con la consecuente formación de microsistemas óptimos para el desarrollo de aquellos hongos que se han introducido asociados a dichas especies ${ }^{3}$.

Se reportan dos nuevos registros de Agaricales para los bosques nativos de Nothofagus de la Patagonia argentina, ambos citados previamente creciendo asociados a especies de interés forestal para la región de Patagonia chilena 4 .

\section{MATERIALES Y MÉTODOS}

Los ejemplares coleccionados fueron analizados tanto macro como microscópicamente de acuerdo a Mata Hidalgo et al. ${ }^{5}$. El mapa fue elaborado usando Quantum GIS (www.qgis. org), los mapas políticos se obtuvieron de DIVA-GIS (www.diva-gis.org/gdata, ${ }^{6}$ ), las capas fueron obtenidas del Inventario Nacional de Bosques Nativos ${ }^{7}$, y fueron agrupadas en dos categorías: la primera, "Bosque Nativo" incluye áreas constituidas por bosques inventariables de lenga, ciprés, coihue y de bosques mixtos. La categoría "Forestaciones" incluye los bosques implantados de pino.
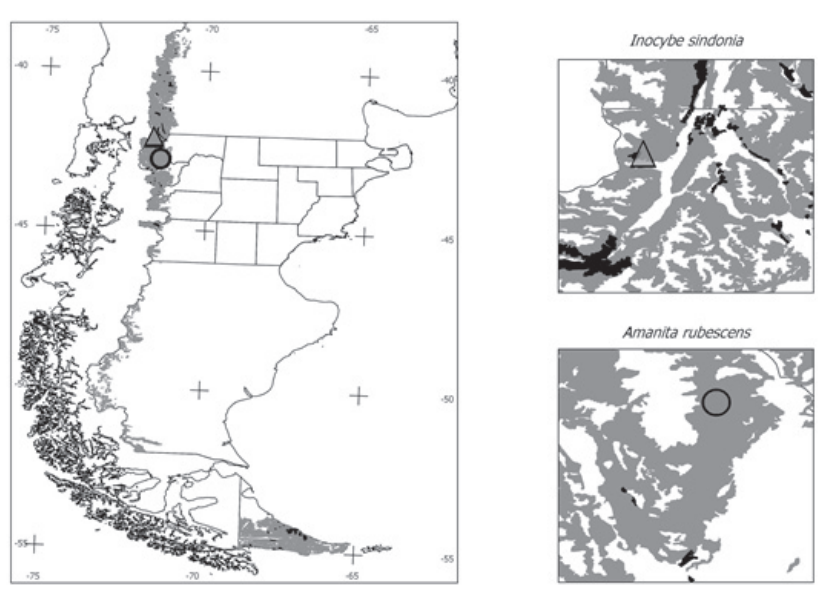

Figura 1. Mapa de sitios de recolección de $I$. sindonia (triángulo) y A. rubescens (círculo) en Chubut, Argentina. Categorías: Bosque nativo (gris), forestaciones (negro).

\section{RESULTADOS}

Inocybe sindonia (Fr.) P. Karst., Bidr. Känn. Finl. Nat. Folk 32: 465 (1879). Fig. 2.

$\equiv$ Agaricus sindonius Fr. 1838 Fr., Epicr. syst. mycol. (Upsaliae): 176 (1838). Descripción. Valenzuela ${ }^{4}$.

Material estudiado.- ARGENTINA. Prov. Chubut, Dpto. Cushamen, Lago Puelo, $42^{\circ}$ 5'45'S 7141'40”O, 240m, IV-2013, Gonzalo M. Romano GR14025.

Notas.- En suelo, bajo Nothofagus dombeyi.

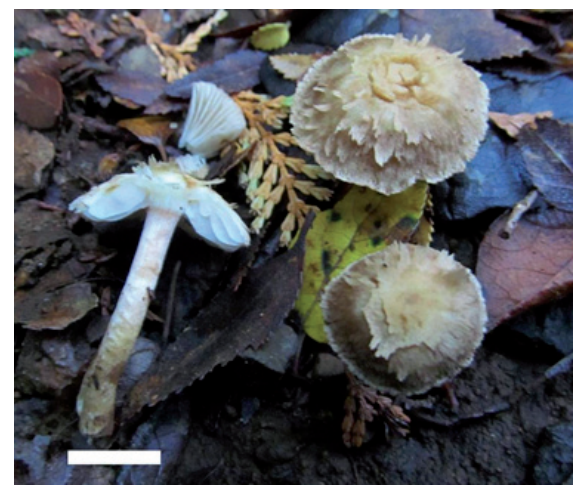

Figura 2. Aspecto general de Inocybe sindonia. Regla: $1 \mathrm{~cm}$. 
Amanita rubescens Pers., Tent. disp. meth. fung. (Lipsiae): 71 (1797). Fig. 3.

Descripción.- Valenzuela ${ }^{4}$.

Material estudiado.- ARGENTINA. Prov. Chubut, Dpto. Futaleufú, Huemules, 42 $46^{\circ} 43^{\prime \prime} \mathrm{S}$ 71²7’48'O, 1140 m, III-2014, Gonzalo M. Romano GR52328.

Notas.- En suelo, bajo Nothofagus pumilio.

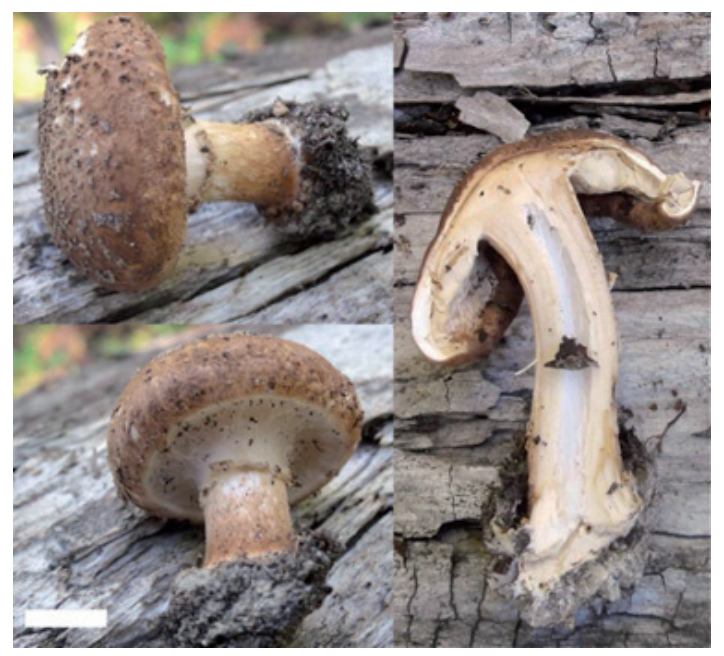

Figura 3. Aspecto general de Amanita rubescens. Regla: $1 \mathrm{~cm}$.

\section{DISCUSIÓN}

Las dos especies de Agaricales aquí citadas constituyen dos nuevos registros de Agaricales para los bosques andino patagónicos de Nothofagus de la Argentina. Inocybe sindonia ha sido descrita por Valenzuela ${ }^{4}$ bajo Pseudotsuga menziesii y Fitzroya cupressoides para los bosques de Chile. El ejemplar hallado en Lago Puelo se encontraba bajo $N$. dombeyi, en una zona cercana a plantaciones de Ps. menziesii.

Amanita rubescens también es una especie micorrícica. Valenzuela ${ }^{4}$ señala que en Chile se la encuentra en bosques de Pinus radiata y también en bosques de $N$. dombeyi (coihue). Se trata de una especie de baja especificidad para formar asociaciones micorrícicas, por lo que posee un amplio potencial para desplazar a algunas especies micorrícicas nativas como las de Cortinarius en los bosques andinos ${ }^{8}$. Esta característica, sumada al crecimiento sostenido de la superficie utilizada para plantaciones forestales favorecen que $A$. rubescens pueda invadir los bosques de las distintas especies de Nothofagus. La zona de bosque de Huemules se encuentra rodeada por diversas plantaciones forestales de $P$. radiata que podrían ser el punto de partida de la invasión de $A$. rubescens.

Inocybe sindonia y A. rubescens poseen la capacidad de formar asociaciones micorrícicas con un amplio espectro de coníferas y caducifolias, a diferencia de aquellas especies nativas de Cortinarius que únicamente pueden asociarse con Nothofagus ${ }^{9}$. Esta baja especificidad para formar micorrizas les otorga una ventaja competitiva que permitiría un comportamiento invasivo desde las plantaciones forestales hacia el bosque nativo, como se muestra en la figura 1 .

Se trata de dos eventos distintos que dejarían en evidencia la alteración de la flora nativa del ecosistema de bosque andino patagónico por el efecto de la introducción de especies forestales exóticas, como P. radiata y Ps. menziesii, con la potencial modificación de la micobiota asociada.

\section{AGRADECIMIENTOS}

Los autores agradecen al equipo de trabajo del Laboratorio de Geomática del CIEFAP, quienes gentilmente nos permitieron acceder a las capas del Inventario Nacional de Bosques Nativos; también al CONICET por financiar el presente trabajo. 


\section{REFERENCIAS}

1. Salomón MES, Barroetaveña C y Rajchenberg M. Do pine plantations provide mycorrhizal inocula for seedlings establishment in grasslands from Patagonia, Argentina?. New forests. 2011; 41(2): 191-205.

2. Bava J. El uso forestal de los bosques de Lenga argentinos y la certificación de manejo del Forest Statewardship Council. Quebracho. 2003; 10: 53-59.

3. Garrido N y Bresinsky A. Amanita merxmuelleri (Agaricales), eine neue Art aus NothofagusWtildern Chiles. Botanische Jahrbücher Systematik. 1985; 107: 521-540.

4. Valenzuela E. Estudio sistemático, corológico y ecológico de los Agaricales sensu lato de los bosques autóctonos de la región de Los Lagos en Chile. Trabajo de doctorado. Departamento de Biología Vegetal. Sección Biológicas. Facultad de Ciencias. Universidad de Alcalá de Henares. España. 1993.348 pp.

5. Mata Hidalgo M, Umaña L y Chaves J. Documento borrador de referencia Protocolo de ma- nejo de colecciones de hongos. INBio Costa Rica \& Norwegian Ministry of Foreign Affairs, 29 p. 2009. Disponible en: http://inbio.ac.cr/web_herbarios/web/pdf/protocolo-hongos.pdf

6. Hijmans RJ, Guarino L, Cruz M y Rojas E. Computer tools for spatial analysis of plant genetic resources data: 1. DIVA-GIS. Plant Genetic Resources Newsletter. 2001; 127: 15-19.

7. Primer Inventario Nacional de Bosques Nativos. Proyecto de Bosques Nativos y Áreas Protegidas. Préstamo BIRF 4085-AR. Informe regional bosque andino patagónico. 2001.

8. Valenzuela E, Moreno G, Garnica S y Ramirez C. Micosociología en bosques nativos de Nothofagus y plantaciones de Pinus radiata en la X Región de Chile: diversidad y rol ecológico. Revista Chilena de Historia Natural. 1998; 71: 133146.

9. Rinaldi AC, Comandini O, Kuyper T. W. Ectomycorrhizal fungal diversity: separating the wheat from the chaff. Fungal Diversity. 2008; 33: 1-45. 